\title{
BMJ Urinary albumin excretion as a marker Open of endothelial dysfunction in migraine sufferers: the HUNT study, Norway
}

\author{
Line M Jacobsen, ${ }^{1}$ Bendik S Winsvold, ${ }^{1,2}$ Solfrid Romundstad, ${ }^{3,4}$ \\ Are H Pripp, ${ }^{5}$ Jostein Holmen, ${ }^{6}$ John-Anker Zwart ${ }^{1,2,7}$
}

To cite: Jacobsen LM, Winsvold BS, Romundstad S, et al. Urinary albumin excretion as a marker of endothelial dysfunction in migraine sufferers: the HUNT study, Norway. BMJ Open 2013;3:e003268.

doi:10.1136/bmjopen-2013003268

- Prepublication history for this paper is available online. To view these files please visit the journal online (http://dx.doi.org/10.1136/ bmjopen-2013-003268).

Received 21 May 2013 Accepted 4 July 2013

For numbered affiliations see end of article.

Correspondence to Dr Line Melå Jacobsen; linemjaco@gmail.com

\section{ABSTRACT}

Objective: To investigate urine albumin leakage as a marker of endothelial dysfunction in migraine patients.

Design: A population-based health study.

Participants: 303 patients with migraine, 1009

patients with non-migraine headache and 5287

headache-free controls.

Outcomes: The association between urine albuminto-creatine ratio (ACR) and headache status was investigated in the Nord-Trøndelag Health Study (HUNT-2). Patients were selected in two strata, based on either (1) self-reported hypertension/diabetes (morbid sample) or (2) a random sample. Analyses were performed using analysis of covariance.

Results: There was no association between headache status and ACR in the study population $(p=0.23$, mean ACR for migraine $1.66,95 \% \mathrm{Cl} 1.31$ to 2.01 , for nonmigraine headache $1.90,95 \% \mathrm{Cl} 1.71$ to 2.09 and for no headache $1.73,95 \% \mathrm{Cl} 1.64$ to 1.81 ) after relevant adjustments. Similarly, no association between headache status and ACR was seen when the analysis was stratified for morbid and random samples, or for migraine with and without aura.

Conclusions: We found no evidence of increased urine albumin leakage in migraine sufferers when compared with headache-free controls. This could indicate that systemic endothelial dysfunction is not a prominent feature of migraine.

\section{INTRODUCTION}

Migraine is a common disabling headache disorder affecting $6-7 \%$ of men and $18 \%$ of women. ${ }^{1}$ It is rated as one of the top 10 most disabling diseases ${ }^{2}{ }^{3}$ and has been estimated to be the most costly neurological disorder to society. ${ }^{4}$ Migraine manifests in headache attacks lasting 4-72 $\mathrm{h}$ characterised by throbbing, pulsating and unilateral headache, often accompanied by nausea, photophobia and phonophobia. ${ }^{5}$ In up to a third of the patients ${ }^{6}$ these attacks may be accompanied by additional neurological aura symptoms separating the disease into migraine with aura (MA) and migraine without aura (MO). ${ }^{5}$

\section{ARTICLE SUMMARY}

Article focus

- We aimed to investigate urine albumin-to-creatine ratio (ACR) as a marker of endothelial dysfunction in patients with migraine, non-migraine headache and headache-free controls.

\section{Key messages}

- No associations were found between headache status and ACR, implying the absence of large differences in urine albumin leakage between migraine patients or other headache sufferers and headache-free controls.

- This may indicate that systemic endothelial dysfunction is not a prominent feature of migraine.

Strengths and limitations of this study

- The study is the first to assess albumin leakage as a marker of endothelial dysfunction in migraine patients.

- The study includes a relatively large sample size, objective measurements of albumin leakage and the use of validated headache diagnoses.

- The sample size might be too low to detect ACR differences among migraine subtypes. In particular, there was a limited number of patients having migraine with aura in this study.

Migraine, in particular MA, has been associated with an increased risk of cardiovascular disease (CVD) including ischaemic stroke, ischaemic lesions of the brain, myocardial infarction, angina and cardiovascular death. ${ }^{7-9}$ While the mechanisms underlying this link remain unknown, multiple explanations have been suggested. Shared environmental or genetic risk factors could be present, changes in vascular function might contribute to migraine pathophysiology or the opposite; migraine pathophysiology could induce changes in vascular function, or mutual mechanistic pathways may exist. ${ }^{10}$

The vascular endothelium plays an important role in setting vascular tone, regulating vascular permeability, maintaining thrombotic 
balance and regulating fibrinolytic and inflammatory pathways. A dysfunctional vascular endothelium is often observed in CVD. ${ }^{11}$ Interestingly, abnormal control of systemic vascular tone, ${ }^{12}$ alterations in systemic arterial structure and function ${ }^{13}$ and impaired ability to repair systemic endothelial injury ${ }^{14}$ have also been reported in migraine patients. The increase of systemic vasoactive mediators during migraine attacks, including the vasoconstrictive factor ET-1 and the pro-inflammatory factor $\mathrm{C}$ reactive protein, suggests an involvement of the endothelium in migraine headache. ${ }^{15}{ }^{16}$ Endothelial dysfunction could also be implicated in the generation of migraine aura as ET-1 has been shown to produce cortical spreading depression, the presumed substrate of migraine aura, in rats. ${ }^{17}$ Furthermore, migraine patients with aura have an increased risk of thrombotic events. It has been reported raised plasma levels of vWf in migraine patients, ${ }^{18}$ a factor that promotes clotting and formation when the endothelium is damaged, further supporting the presence of endothelial dysfunction in migraine. This could explain the link between migraine and CVD and might elucidate pathophysiological mechanisms of migraine.

The presence of subclinical increases in urinary albumin excretion (UAE) has been associated with impaired endothelium-dependent vasodilation ${ }^{19}$ and is believed to reflect endothelial dysfunction. ${ }^{20}{ }^{21}$ No previous studies have, however, investigated albumin excretion in migraine patients. The aim of this study was therefore to compare urine albumin-to-creatine ratio (ACR), as a measure of UAE, in patients with migraine, non-migraine headache and headache-free controls.

\section{METHODS}

\section{Participants and headache diagnoses}

Participants were selected from the second Nord-Trøndelag Health Study (HUNT 2), a general health survey conducted in 1995-1997. All inhabitants aged $\geq 20$ years residing in the county at the time of screening were invited $(n=92703)$ and 65258 patients $(70.4 \%)$ participated. ${ }^{22}$ The population in Nord-Trøndelag County is ethnically homogenous with less than 3\% non-Caucasians. All participants were mailed a questionnaire and attended a clinical examination (for details see ref. 22). The questionnaire included questions on previous or current CVD, hypertension, diabetes mellitus and smoking habits as well as 13 headache questions. The clinical examination included measurements of height, weight and blood pressure. When attending the health examination, participants received a second questionnaire to complete and return by mail. The 13 headache questions of the questionnaire were designed mainly to determine whether the individual had headache, the frequency of headache, and to diagnose migraine according to a modified version of the ICHD migraine criteria. ${ }^{23}{ }^{24}$ Participants who answered 'yes' to the question 'Have you suffered from headache during the last 12 months?' were classified as headache sufferers. Those who answered 'no' comprised the headache-free control group. Based on the subsequent 12 headache questions, headache sufferers were classified as having migraine if they fulfilled the following three criteria: (1) headache attacks lasting 4-72 $\mathrm{h}$ (less than $4 \mathrm{~h}$ was accepted for those who reported often visual disturbances before headache); (2) headache with at least one of the following characteristics: pulsating quality, unilateral location, aggravation by physical activity; (3) during headache, at least one of the following: nausea, photophobia or phonophobia. Those within this group who reported often having visual disturbance prior to headache were classified as having MA. Headache sufferers who did not fulfil the criteria for migraine and did not have self-reported migraine were classified as having non-migraine headache. Headache diagnoses were mutually exclusive. The headache diagnoses were validated by clinical interviews by physicians with long clinical experience in headache disorders. The positive and negative predictive values of the questionnaire-based diagnoses were, respectively, $68 \%$ and $76 \%$ for non-migraine headache, $87 \%$ and $75 \%$ for migraine, and $100 \%$ and $91 \%$ for MA. A total of 9598 participants were included in a further study in which they donated three urine samples for the determination of ACR, originally designed for investigating UAE in hypertension and diabetes. Participants were included based on (1) the presence of self-reported diabetes mellitus and/or medically treated hypertension (morbid sample) and (2) a 5\% randomly selected sample of the total population (random sample). Participants who contributed with information regarding both headache status and ACR were eligible for the present study. Participants with overt proteinuria, ACR $\geq 25 \mathrm{mg} / \mathrm{mmol}$ in men and $\geq 35 \mathrm{mg} / \mathrm{mmol}$ in women, one participant with migraine (with aura), 16 participants with non-migraine headache and 141 participants without headache, were excluded. In total, 303 participants with migraine, 1009 participants with non-migraine headache and 5287 headache-free participants were included in the study, in which the morbid sample constituted 3688 medically treated hypertensive participants, 647 diabetic participants and 413 participants with both medically treated hypertension and diabetes mellitus.

\section{Urine sampling}

Participants received a unit with three plastic receptacles for three first morning urine samples, three transport tubes and one envelope for returning the samples by mail to the laboratory. Of 11661 packs handed out, $9598(82.3 \%)$ participants mailed three samples back to the laboratory. ${ }^{25}$ Those who failed to return three samples were excluded. A written instruction describing how to collect urine, information about the screening and a questionnaire concerning urinary tract infection in the previous week, persistent haematuria in the last year and menstruation at the time of collection was included. While the most consistent method for determining UAE is $24 \mathrm{~h}$ urine sampling, it has been shown 
that measuring ACR in one or more morning spot urine samples provides good specificity and sensitivity. ${ }^{26}$

\section{Laboratory procedures}

Fresh, non-frozen urine samples were analysed at the Central Laboratory at Levanger Hospital on a Hitachi 91 Autoanalyzer (Hitachi, Mito, Japan). Urine albumin and creatine levels were determined using an immunoturbimetric method (antihuman serum albumin; Dako Norway, Oslo) and Jaffé method, respectively. The ACR, measured in $\mathrm{mg} / \mathrm{mmol}$, was used as an expression of UAE.

\section{Consent}

Participation in HUNT2 was voluntary and each participant signed a written consent. All surveys and analyses were approved by the Norwegian Data Inspectorate and by the Regional Committee for Medical and Health Research Ethics.

\section{Statistical analysis}

For all analyses we used mean ACR from three delivered urine samples, measured in $\mathrm{mg} / \mathrm{mmol}$. Comparisons of mean ACR between cases with migraine, non-migraine headache and headache-free controls in the morbid, random and combined samples were performed by analysis of covariance. The confounding effect of potential confounders, that is, age, sex, body mass index (BMI), smoking, self-reported diabetes and self-reported antihypertensive medication, in the combined sample was calculated by the formula (mean $\mathrm{ACR}_{\text {crude }}$-mean $\mathrm{ACR}_{\mathrm{adj}}$ )/ mean $\mathrm{ACR}_{\text {crude, }}$ where mean $\mathrm{ACR}_{\text {crude }}$ was the mean ACR in the crude model and mean $A_{C R}$ adj the mean ACR in the model adjusted for the relevant variable. Variables with a confounding effect $>5 \%$ in one or more headache group were included in the final model. A p value less than 0.05 was used to indicate statistical significance.

\section{RESULTS}

Baseline characteristics for the random and morbid samples are shown in table 1. The morbid sample was characterised by a higher age and BMI and a lower proportion of smokers as compared with the random sample. For both samples a higher proportion of women had headache than men, with the highest percentage of women found among the migraine participants. The mean age was lower in the headache groups than in controls.

Crude analyses indicated an association between ACR and headache status in the morbid sample $(p=0.013$, mean ACR for migraine 1.22, for non-migraine headache 1.95 and for no headache 2.03) and the random sample ( $\mathrm{p}=0.011$, mean ACR for migraine 0.85 , for non-migraine headache 0.96 and for no headache 1.22) (table 2). However, when adjusting for age and sex this effect disappeared (model I). No significant associations were observed between headache status and ACR in the morbid sample $(p=0.11$, mean ACR for migraine 1.87 , for non-migraine headache 2.27 and for no headache 1.96) or the random sample $(p=0.55$, mean ACR for migraine 1.00, for non-migraine headache 1.07 and for no headache 1.16) when adjusted for age, sex, selfreported diabetes and self-reported use of antihypertensive medication (model II).

A similar pattern was observed when morbid and random samples were combined; crude data indicated an association between ACR and headache status, but this effect was explained by differences in age and sex, table 3. No significant associations were observed when adjusted for age, sex, self-reported diabetes and selfreported use of antihypertensive medication $(p=0.23$, mean ACR for migraine 1.66, for non-migraine headache 1.90 and for no headache 1.73). Mean differences for ACR in the combined sample (model II) were -0.07 (95\% CI -0.43 to 0.30$)$ between migraine participants and headache-free controls and 0.18 (95\% CI -0.04 to 0.39 ) between non-migraine headache and headachefree controls. We also examined the association between

\begin{tabular}{|c|c|c|c|}
\hline & No headache & Non-migraine headache & Migraine \\
\hline \multicolumn{4}{|l|}{ Morbid sample $(n=4748)$} \\
\hline Women (\%) & 51.7 & 60.2 & 69.8 \\
\hline Mean age (SD) & $67.0(11.2)$ & $58.7(12.9)$ & $51.5(12.5)$ \\
\hline Antihypertensive use (\%) & 86.9 & 84.4 & 83.0 \\
\hline Diabetes (\%) & 22.5 & 22.5 & 20.9 \\
\hline Daily smokers (\%) & 19.3 & 24.6 & 23.1 \\
\hline Mean body mass index (SD) & $28.6(4.5)$ & $29.0(4.9)$ & $28.7(4.8)$ \\
\hline \multicolumn{4}{|l|}{ Random sample $(n=1851)$} \\
\hline Women (\%) & 46.8 & 63.2 & 75.0 \\
\hline Mean age (SD) & $53.9(16.5)$ & $43.6(13.7)$ & $39.6(11.1)$ \\
\hline Antihypertensive use (\%) & 13.1 & 7.2 & 7.0 \\
\hline Diabetes (\%) & 4.1 & 1.2 & 0.0 \\
\hline Daily smokers (\%) & 27.3 & 33.3 & 34.8 \\
\hline Mean body mass index (SD) & $26.5(4.0)$ & $26.1(4.0)$ & $25.8(3.8)$ \\
\hline
\end{tabular}


Table 2 Albumin-to-creatine ratio (ACR) by headache status and selection criterium

\begin{tabular}{|c|c|c|c|c|c|c|}
\hline & \multicolumn{2}{|l|}{ Crude } & \multicolumn{2}{|l|}{ Model I‡ } & \multicolumn{2}{|l|}{ Model II§ } \\
\hline & Mean ACR (95\% Cl) N & p Value & Mean ACR (95\% Cl) N & p Value & Mean ACR (95\% Cl) N & p Value \\
\hline Morbid sample & & $0.013^{*}$ & & $0.13^{*}$ & & $0.11^{*}$ \\
\hline No headache & 2.03 (1.92 to 2.14 ) 3998 & & 1.96 (1.85 to 2.07$) 3998$ & & 1.96 (1.85 to 2.06$) 3978$ & \\
\hline Non-migraine headache & 1.95 (1.68 to 2.23 ) 591 & $0.61 \dagger$ & 2.26 (1.97 to 2.54) 591 & $0.057 \dagger$ & 2.27 (1.99 to 2.55 ) 585 & $0.044 \dagger$ \\
\hline Migraine & 1.22 (0.68 to 1.75$) 159$ & $0.003+$ & 1.85 (1.31 to 2.40$) 159$ & $0.70 \dagger$ & 1.87 (1.33 to 2.41$) 158$ & $0.75 \dagger$ \\
\hline Random sample & & $0.011^{*}$ & & $0.60^{*}$ & & $0.55^{\star}$ \\
\hline No headache & 1.22 (1.11 to 1.32$) 1289$ & & 1.16 (1.06 to 1.27$) 1289$ & & 1.16 (1.05 to 1.26$) 1285$ & \\
\hline Non-migraine headache & $0.96(0.77$ to 1.14$) 418$ & $0.017 \dagger$ & 1.08 (0.89 to 1.26$) 418$ & $0.44 \dagger$ & 1.07 (0.89 to 1.26$) 417$ & $0.44 \dagger$ \\
\hline Migraine & 0.85 (0.53 to 1.16$) 144$ & $0.028 \dagger$ & $1.02(0.70$ to 1.34$) 144$ & $0.41 \dagger$ & 1.00 (0.68 to 1.31$) 143$ & $0.35 \dagger$ \\
\hline
\end{tabular}

${ }^{*} p$ Value for overall effect of headache groups.

tp Values are for comparison against no headache group within the same selection criterium.

$\ddagger$ Model I: adjusted for age and sex.

$\S$ Model II: adjusted for age, sex, self-reported diabetes and self-reported use of antihypertensive medication.

Table 3 Albumin-to-creatine ratio (ACR) by headache status in combined sample

\begin{tabular}{|c|c|c|c|c|c|c|}
\hline & \multicolumn{2}{|l|}{ Crude } & \multicolumn{2}{|l|}{ Model I¥ } & \multicolumn{2}{|l|}{ Model II§ } \\
\hline & $\overline{\text { Mean ACR (95\% Cl) N }}$ & p Value & Mean ACR (95\% Cl) N & p Value & Mean ACR (95\% CI) N & p Value \\
\hline & & $<0.001^{*}$ & & $0.29^{*}$ & & $0.23^{*}$ \\
\hline No headache & 1.83 (1.75 to 1.92$) 5287$ & & 1.73 (1.65 to 1.81$) 5287$ & & 1.73 (1.64 to 1.81$) 5263$ & \\
\hline Non-migraine headache & 1.54 (1.35 to 1.73$) 1009$ & $0.007 \dagger$ & 1.89 (1.69 to 2.09) 1009 & 0.15 & 1.90 (1.71 to 2.09) 1002 & $0.11 \dagger$ \\
\hline Migraine & 1.04 (0.69 to 1.39$) 303$ & $<0.001 \dagger$ & 1.67 (1.31 to 2.03$) 303$ & 0.74 & 1.66 (1.31 to 2.01$) 301$ & $0.72 \dagger$ \\
\hline
\end{tabular}

*p Value for overall effect of headache groups.

tp Values are for comparison against no headache group.

$\neq$ Model I: adjusted for age and sex.

§Model II: adjusted for age, sex, self-reported diabetes and self-reported use of antihypertensive medication. 
headache status and ACR after stratification of migraine into its two subtypes MA and MO. In the adjusted model, no differences in ACR scores were found between migraine subtype and controls $(\mathrm{p}=0.68$, mean ACR for MA 1.36, for MO 1.73 and for no headache 1.73; table 4). There was no interaction between headache status and self-reported diabetes, or between headache status and self-reported use of antihypertensive medication on ACR. Since the measurement ACR was positively skewed, we repeated the analyses after square root transformation, which provided similar results. To ease interpretation only non-transformed results are presented.

\section{DISCUSSION}

The present study did not find an association between headache status and ACR, indicating the absence of large differences in urine albumin leakage between migraine participants or other headache sufferers and headache-free controls. The mechanisms linking migraine and CVD are likely complex, but dysregulation of vascular endothelium as expressed by albumin leakage is not supported by the present data to be a major factor.

The study includes a relatively large sample size, objective measurements of UAE, analyses based on fresh urine samples and the use of validated headache diagnoses. Furthermore, the design of the study allowed us to evaluate the association between headache status and UAE in a random sample as well as a morbid sample, which included a high proportion of participants with hypertension and diabetes types I and II, well-known risk factors for increased UAE. ${ }^{27-29}$ A limitation of the study is, however, that hypertension and diabetes information was based on self-report. Data on participants' migraine status (ictal or interictal phase) during urine sampling was not available and it cannot be excluded that there may be a difference in urine albumin leakage during and outside a migraine attack. In addition, the sample size might be too low to detect ACR differences among migraine subtypes. In particular, there was a limited number of participants having MA in this study.

A high level of urine ACR, most likely representing excessive glomerular capillary leakage of albumin, ${ }^{19}$ is a risk marker for cardiovascular morbidity and mortality ${ }^{30}$ and is believed to reflect endothelial dysfunction. ${ }^{19} 21$ Increased UAE has been associated with impaired endothelium-dependent vasodilatation, ${ }^{19}$ impaired peripheral vasoreactivity to endothelial agonists ${ }^{31}$ as well as with circulating biomarkers of endothelial dysfunction. ${ }^{32}$ Abnormal ACR levels in urine have also been reported in inflammatory, non-cardiovascular conditions such as rheumatoid arthritis, inflammatory bowel syndrome and surgery, which has been hypothesised to be a result of circulating inflammatory molecules damaging parenchymal functions of the kidneys. ${ }^{20}$ Interestingly, ACR levels even within the normal range have been associated with

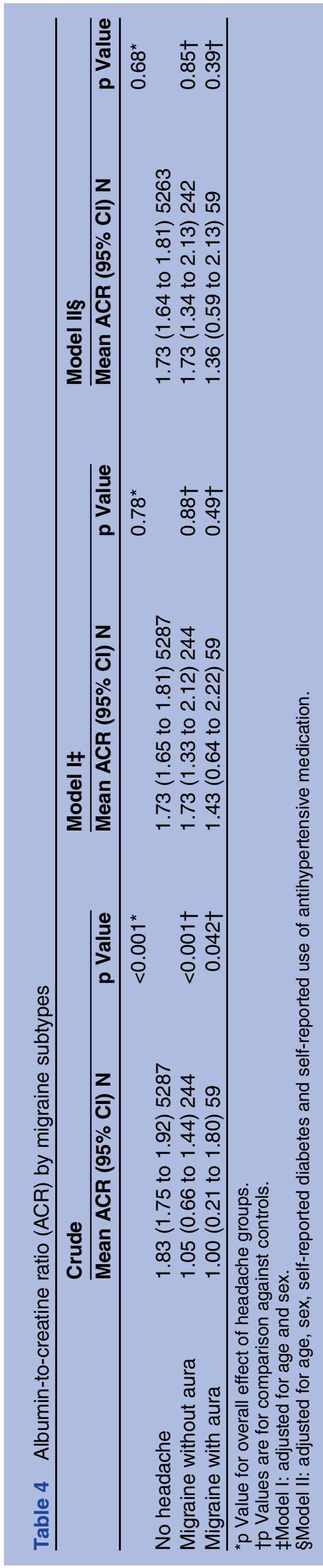


an increased rate of kidney disease, CVD and mortality. ${ }^{33-35}$ As UAE is a biological continuum, we used continuous ACR values in the present study rather than applying microalbuminuria commonly defined as ACR $\geq 2.5 \mathrm{mg} / \mathrm{mmol}$. The mean ACR levels in the morbid group of the present study were twice as high as for the randomly chosen sample, which is explained by the presence of hypertension and diabetes. In the random sample, however, ACR was generally low for all headache and headache-free groups.

The unadjusted analysis indicated an association between ACR and headache status in the morbid, random and combined samples. However, when adjusting for age and sex the association disappeared, suggesting these variables to be the major contributing factors for differences in ACR in the present study. Given the fact that women have less muscular mass and lower creatine excretion than men, and that creatine excretion decreases with age, ${ }^{25}$ a confounding effect of age and sex on ACR was not unexpected.

Contrary to our hypothesis we did not find urine albumin leakage to be more prominent among migraine sufferers than among headache-free participants. If endothelial dysfunction with albumin leakage was a feature of migraine, we would expect elevated ACR levels among migraine participants, and highest among MA. Although we could not find an association between ACR and migraine status, there was a non-significant tendency in the opposite direction; participants with MA had the lowest ACR levels. While many previous studies favour the presence of endothelial dysfunction in migraine patients, some studies argue against it. In fact, systemic endothelium-dependent vasodilation, nitric oxide levels and stimulated t-PA levels have been found similarly expressed in migraine participants and healthy controls. ${ }^{36-38}$ Furthermore, it was recently suggested that extracranial dilatation may not be relevant for migraine pain during attacks. ${ }^{39}$

In summary, in this first study examining albumin leakage in migraine sufferers, we did not find urine albumin leakage to be more prominent among migraine participants than among headache-free participants, which could indicate that systemic endothelial dysfunction is not a prominent feature of migraine.

\section{Author affiliations \\ ${ }^{1}$ FORMI, Oslo University Hospital, Oslo, Norway \\ ${ }^{2}$ Department of Neurology, Oslo University Hospital, Oslo, Norway \\ ${ }^{3}$ Department of Cancer Research and Molecular Medicine, Norwegian \\ University of Science and Technology, Trondheim, Norway \\ ${ }^{4}$ Department of Internal Medicine, Levanger Hospital, Health Trust Nord- Trøndelag, Levanger, Norway \\ ${ }^{5}$ Department of Biostatistics, Epidemiology and Health Economy, Oslo University Hospital, Oslo, Norway \\ ${ }^{6}$ Department of Public Health and General Practice, HUNT Research Centre, Norwegian University of Science and Technology, Levanger, Norway ${ }^{7}$ Institute of Clinical Medicine, University of Oslo, Norway}

Contributors LMJ contributed to the study design, data analysis, data interpretations and wrote the manuscript. BSW contributed to the data analysis, data interpretations and the drafting of the manuscript. SR and $\mathrm{JH}$ conceived the original study, provided laboratory resources and contributed to the manuscript. AHP contributed to the data analysis, data interpretations and the manuscript. JAZ contributed to the study design, data interpretations to the manuscript. All authors approved the final version of the manuscript.

Funding The present study was funded by South-Eastern Norway Regional Health Authority, which had no role in the design or conduct of the study. The Nord-Trøndelag Health Study (HUNT) is a collaboration between HUNT Research Centre, Faculty of Medicine, Norwegian University of Science and Technology (NTNU, Levanger), Norwegian Institute of Public Health and Nord-Trøndelag County Council.

Competing interests None.

Ethics approval The Norwegian Data Inspectorate and the Regional Committee for Medical and Health Research Ethics approved the study.

Provenance and peer review Not commissioned; externally peer reviewed.

Data sharing statement No additional data are available.

Open Access This is an Open Access article distributed in accordance with the Creative Commons Attribution Non Commercial (CC BY-NC 3.0) license, which permits others to distribute, remix, adapt, build upon this work noncommercially, and license their derivative works on different terms, provided the original work is properly cited and the use is non-commercial. See: http:// creativecommons.org/licenses/by-nc/3.0/

\section{REFERENCES}

1. Stovner LJ, Andree C. Prevalence of headache in Europe: a review for the Eurolight project. J Headache Pain 2010;11:289-99.

2. Vos $T$, Flaxman AD, Naghavi M, et al. Years lived with disability (YLDs) for 1160 sequelae of 289 diseases and injuries 1990-2010: a systematic analysis for the Global Burden of Disease Study 2010. Lancet 2012;380:2163-96.

3. Steiner TJ, Stovner LJ, Birbeck GL. Migraine: the seventh disabler. Headache 2013:53:227-9.

4. Andlin-Sobocki $\mathrm{P}$, Jonsson $\mathrm{B}$, Wittchen $\mathrm{HU}$, et al. Cost of disorders of the brain in Europe. Eur J Neurol 2005;12(Suppl 1):1-27.

5. Headache Classification Subcommittee of the International Headache S. The International Classification of Headache Disorders: 2nd edition. Cephalalgia 2004;24(Suppl 1):9-160.

6. Lipton RB, Stewart WF, Diamond S, et al. Prevalence and burden of migraine in the United States: data from the American Migraine Study II. Headache 2001;41:646-57.

7. Schurks M, Rist PM, Bigal ME, et al. Migraine and cardiovascular disease: systematic review and meta-analysis. BMJ 2009;339:b3914.

8. Kruit MC, van Buchem MA, Hofman PA, et al. Migraine as a risk factor for subclinical brain lesions. JAMA 2004;291:427-34.

9. Gudmundsson LS, Scher AI, Aspelund T, et al. Migraine with aura and risk of cardiovascular and all cause mortality in men and women: prospective cohort study. BMJ 2010;341:c3966.

10. Bigal ME, Kurth $\mathrm{T}, \mathrm{Hu} \mathrm{H}$, et al. Migraine and cardiovascular disease: possible mechanisms of interaction. Neurology 2009;72:1864-71.

11. Cai H, Harrison DG. Endothelial dysfunction in cardiovascular diseases: the role of oxidant stress. Circ Res 2000;87:840-4.

12. Schwedt TJ. Endothelial dysfunction in migraine. Cephalalgia 2009;29:997-1002.

13. Yetkin $\mathrm{E}$, Ozisik $\mathrm{H}$, Ozcan $\mathrm{C}$, et al. Increased dilator response to nitrate and decreased flow-mediated dilatation in migraineurs. Headache 2007;47:104-10.

14. Lee ST, Chu K, Jung KH, et al. Decreased number and function of endothelial progenitor cells in patients with migraine. Neurology 2008;70:1510-17.

15. Gallai V, Sarchielli P, Firenze C, et al. Endothelin 1 in migraine and tension-type headache. Acta Neurol Scand 1994;89:47-55.

16. Tietjen GE, Herial NA, White $L$, et al. Migraine and biomarkers of endothelial activation in young women. Stroke 2009;40:2977-82.

17. Dreier JP, Kleeberg J, Petzold G, et al. Endothelin-1 potently induces Leao's cortical spreading depression in vivo in the rat: a model for an endothelial trigger of migrainous aura? Brain 2002;125 (Pt 1):102-12.

18. Tietjen GE, Al-Qasmi MM, Athanas K, et al. Increased von Willebrand factor in migraine. Neurology 2001;57:334-6.

19. Malik A, Sultan S, Turner S, et al. Urinary albumin excretion is associated with impaired flow- and nitroglycerin-mediated brachial 
artery dilatation in hypertensive adults. $J$ Hum Hypertens 2007;21:231-8

20. Pedrinelli R, Dell'Omo G, Penno G, et al. Non-diabetic microalbuminuria, endothelial dysfunction and cardiovascular disease. Vasc Med 2001;6:257-64.

21. Naidoo D. The link between microalbuminuria, endothelial dysfunction and cardiovascular disease in diabetes. Cardiovasc J S Afr 2002;13:194-9.

22. Holmen J, Midthjell K, Krüger $\varnothing$, et al. The Nord-Trøndelag Health Study 1995-97 (HUNT2): objectives, contents, methods and participation. Norsk Epidemiologi 2003;13:19-32.

23. Hagen K, Zwart JA, Vatten L, et al. Head-HUNT: validity and reliability of a headache questionnaire in a large population-based study in Norway. Cephalalgia 2000;20:244-51.

24. Headache Classification Committee of the International Headache Society. Classification and diagnostic criteria for headache disorders, cranial neuralgias and facial pain. Cephalalgia 1988;8 (Suppl 7):1-96.

25. Hallan $\mathrm{H}$, Romundstad S, Kvenild $\mathrm{K}$, et al. Microalbuminuria in diabetic and hypertensive patients and the general populationconsequences of various diagnostic criteria-the Nord-Trondelag Health Study (HUNT). Scand J Urol Nephrol 2003;37:151-8.

26. Jensen JS, Clausen P, Borch-Johnsen K, et al. Detecting microalbuminuria by urinary albumin/creatinine concentration ratio. Nephrol Dial Transplant 1997;12(Suppl 2):6-9.

27. Viberti GC, Hill RD, Jarrett RJ, et al. Microalbuminuria as a predictor of clinical nephropathy in insulin-dependent diabetes mellitus. Lancet 1982;1:1430-2.

28. Diener HC, Kurth T. Is migraine a risk factor for stroke? Neurology 2005:64:1496-7.

29. Cerasola G, Cottone S, Mule G, et al. Microalbuminuria, renal dysfunction and cardiovascular complication in essential hypertension. J Hypertens 1996;14:915-20.
30. Kuritzky L, Toto R, Van Buren P. Identification and management of albuminuria in the primary care setting. $J$ Clin Hypertens (Greenwich) 2011;13:438-49.

31. Perticone F, Maio R, Tripepi G, et al. Endothelial dysfunction and mild renal insufficiency in essential hypertension. Circulation 2004;110:821-5.

32. Pedrinelli R, Giampietro O, Carmassi F, et al. Microalbuminuria and endothelial dysfunction in essential hypertension. Lancet 1994;344:14-18.

33. Romundstad S, Holmen J, Kvenild K, et al. Microalbuminuria and all-cause mortality in 2,089 apparently healthy individuals: a 4.4-yea follow-up study. The Nord-Trondelag Health Study (HUNT), Norway. Am J Kidney Dis 2003;42:466-73.

34. Chronic Kidney Disease Prognosis CMatsushita K, van der Velde M, et al. Association of estimated glomerular filtration rate and albuminuria with all-cause and cardiovascular mortality in general population cohorts: a collaborative meta-analysis. Lancet 2010;375:2073-81.

35. Klausen K, Borch-Johnsen K, Feldt-Rasmussen B, et al. Very low levels of microalbuminuria are associated with increased risk of coronary heart disease and death independently of renal function, hypertension, and diabetes. Circulation 2004;110:32-5.

36. Vanmolkot $\mathrm{FH}$, de Hoon JN. Endothelial function in migraine: a cross-sectional study. BMC Neurol 2010;10:119.

37. Silva FA, Rueda-Clausen CF, Silva SY, et al. Endothelial function in patients with migraine during the interictal period. Headache 2007;47:45-51.

38. Perko D, Pretnar-Oblak J, Sabovic M, et al. Endothelium-dependent vasodilatation in migraine patients. Cephalalgia 2011;31:654-60.

39. Amin FM, Asghar MS, Hougaard A, et al. Magnetic resonance angiography of intracranial and extracranial arteries in patients with spontaneous migraine without aura: a cross-sectional study. Lancet Neurol 2013;12:454-61. 